\title{
LA PLANEACIÓN POR ESCENARIOS APLICADA SOBRE POLÍTICAS URBANAS: EL CASO DEL MERCADO CENTRAL DE CARTAGENA (COLOMBIA)*
}

\author{
JUAN CARLOS VERGARA-SCHMALBACH**, TOMÁS FONTALVO HERRERA*** \& JOSÉ MORELOS GÓMEZ ${ }^{* * * *}$ \\ UNIVERSIDAD DE CARTAGENA
}

Recibido/ Received/ Recebido: 25/06/2013 - Aceptado/ Accepted / Aprovado: 18/02/2014

\begin{abstract}
Resumen
El mercado central de Cartagena (Colombia), también conocido como Bazurto, es considerado actualmente como un foco de contaminación y caos urbano, siendo a su vez proveedor de recursos alimentarios para cerca del $80 \%$ de la población de la ciudad. Los problemas que aquejan a los habitantes del sector, parecen ser constantes en el tiempo; desde la creación de la primera gran plaza pública de la ciudad en 1904 (conocida como el mercado de Getsemaní), los iterados factores críticos (medio ambiente, economía y sociedad) han transcendido a convertirse en amenazas latentes. Este documento con la aplicación del método de planeación por escenario apoyado en un panel de expertos, visualiza futuros posibles (deseados y proyectados) para el mercado de Bazurto para un periodo de 15 años, en donde el traslado y la intervención, parecen ser las opciones más adecuadas para la solución de dichas problemáticas.

Palabras clave: Políticas públicas, Planeación por escenarios, Plaza de mercado.
\end{abstract}

\section{PLANNING THROUGH SCENARIOS IMPLEMENTED FOR URBAN POLICY: CENTRAL MARKET CASE IN CARTAGENA (COLOMBIA)}

\begin{abstract}
The Cartagena's (Colombia) central market, also known as Bazurto, is currently considered as a pollution source and urban chaos, but at the same time is a provider of food resources for about $80 \%$ of city population. The problems that nearby residents have seem to be permanent over time; since the creation of the first major public town square in 1904 (known as the market of Gethsemane), the ite-
\end{abstract}

Artículo derivado del proyecto de investigación: Planeación por escenarios para el mercado de Bazurto de la ciudad de Cartagena, para un horizonte de tiempo de 15 años, financiado por la Vicerrectoría de Investigación de la Universidad de Cartagena, $2010-2011$.

* Docente de tiempo completo de la Universidad de Cartagena. Ingeniero Industrial (Universidad Tecnológica de Bolívar), Especialista en Finanzas (Universidad de Cartagena), Magister en Administración (Universidad Nacional de Colombia), Candidato a Doctor en Ciencias Sociales mención Gerencia (Universidad de Zulia), Investigador del Grupo Métodos Cuantitativos de Gestión de la Universidad de Cartagena. Correo electrónico: jvergaras@unicartagena.edu.co.

*** Docente de tiempo completo de la Universidad de Cartagena. Ingeniero Industrial, Ingeniero Químico, Magister en Administración (Universidad Nacional de Colombia), Candidato a Doctor en Ciencias Sociales mención Gerencia (Universidad de Zulia), Investigador del Grupo Calidad y Productividad Organizacional Integral de la Universidad de Cartagena. Correo electrónico: tfontalvoh@ unicartagena.edu.co.

***** Docente de tiempo completo de la Universidad de Cartagena. Ingeniero Industrial (Universidad Tecnológica de Bolívar), Magister en Administración (Universidad Nacional de Colombia), Candidato a Doctor en Ciencias Sociales mención Gerencia (Universidad de Zulia), Investigador del Grupo Calidad y Productividad Organizacional Integral de la Universidad de Cartagena. Correo electrónico: jmorelosg@unicartagena.edu.co. 
rated critical factors (environment, economy and society) have transcended to become latent threats. This paper based on the implementation of the planning through scenarios approach supported by an experts panel, visualized possible futures (desired and projected) for Bazurto market for a 15 year period where transfer and intervention seem to be the best suitable option for solving such problems.

Keywords: Public policies, Planning through scenarios, Market Square.

\title{
O PLANEJAMENTO POR CENÁRIOS APLICADA A POLÍTICAS URBANAS: O CASO DO MERCADO CENTRAL DE CARTAGENA (COLÔMBIA)
}

\begin{abstract}
Resumo
O mercado central de Cartagena (Colômbia), também conhecido como Bazurto, é considerado atualmente como um foco de contaminação e de caos urbano, sendo por sua vez provedor de recursos alimentícios para aproximadamente $80 \%$ da população da cidade. Os problemas que sofrem os habitantes do setor parecem ser constantes no tempo; desde a criação da primeira grande praça de mercado público da cidade, em 1904 (conhecida como o mercado de Getsêmani), os conhecidos fatores críticos (meio ambiente, economia e sociedade) têm a tendência a se tornarem ameaças latentes. Este documento, com a aplicação do método de planejamento por cenário apoiado num painel de peritos, vislumbra possíveis futuros (desejados e projetados) para o mercado de Bazurto para um período de 15 anos, onde o traslado e a intervenção parecem ser as opções mais adequadas para a solução de ditas problemáticas
\end{abstract}

Palavras chave: Políticas públicas, Planejamento por cenários, Praça de mercado.

Vergara, J., Fontalvo, T. \& Morelos, J. (2014) La Planeación por Escenarios aplicada sobre Políticas Urbanas: El Mercado Central de Cartagena (Colombia). En: Revista de la Facultad de Ciencias Económicas de la Universidad Militar Nueva Granada. rev.fac.cienc.econ, XXII (1)

JEL: R11, R39, R59.

\section{Introducción}

Los estudios relacionados con la visualización del futuro de algo (personas, sociedades, empresas, regiones, naciones) están encaminados a explorar eventos posibles, probables y/o preferibles (Ogilvy \& Schwartz, 1998; Godet, 2000; Lindgren \& Bandhold, 2003; Börjeson et al., 2006). En este sentido, el diseño de escenarios se puede considerar como uno de los métodos disponibles, entre muchos que existen, para prever el futuro (Vergara-Schmalbach et al. 2012a). Por lo general, la literatura relacionada asocia la palabra escenarios con planeación, pensamiento, pronósticos, análisis y aprendizaje (Bradfiel et al., 2005), donde el limitado conocimiento en esta área de la planeación estratégica conlleva al desarrollo de definiciones y metodolo- gías ambiguas, con una aplicación aún escaza en las organizaciones (Wack, 1985; Vergara-Schmalbach et al. 2010).

Actualmente, es inapropiado concebir un método definitivo y eficaz para anticiparse al futuro en ámbito de la gestión pública, ya que existen condiciones de incertidumbre (compuesta por eventos aleatorios) que hacen de las proyecciones, pronósticos, simulaciones y demás métodos prospectivos, funcionen solo como herramientas que intentan revelar el posible devenir. No obstante, poder anticiparse a las oportunidades y amenazas, es una idea que siempre ha estado en la mente de los estrategas. Este conocimiento supondría, anticiparse con preparativos con el fin de sortear las dificultades y aprovechar las oportunidades en el futuro. 
El presente artículo propone en sus resultados, el delineamiento de posibles acciones conducentes a tratar la problemática del mercado de Bazurto (mercado central de la ciudad de Cartagena de Indias, Colombia), relacionado con el impacto negativo que actualmente está causando a los habitantes de la ciudad, a partir de la aplicación del método de planeación por escenarios (en una adaptación del método $\mathrm{SEARCH}$ ) a nivel de gobierno distrital. Como primer aparte de este documento, se incluye una revisión de la literatura y una aproximación al contexto y problemática del mercado de Bazurto, haciendo énfasis en la planeación por escenarios y los factores clave considerados en este estudio. De manera consecuente, se muestra el desarrollo del método SEARCH, los escenarios resultantes (una vez aplicado el método), finalizando en la discusión y conclusiones del caso propuesto.

\section{Revisión de la literatura}

Desde su origen como concepción militar, la planeación por escenarios (que emplea el diseño de escenarios como parte de los procesos de planeación a largo plazo) se ha consolidado como una herramienta organizacional valida y probada, que encaja dentro de cualquier proceso estratégico (Chermac et al., 2001). Los escenarios conducen a la descripción de una imagen probable o posible del futuro, trazando el camino que podría conducir a su consecución (Cornelius et al., 2005; Godet, 2006; Porter, 1998) y que explora el efecto conjunto de varios eventos (Schoemaker, 1995). Con el diseño de escenarios se podrán reducir los riegos, anticipar obstáculos, orientar los planes estratégicos (Vergara-Schmalbach, Máza, \& Fontalvo, 2010) y optimizar el uso de los recursos disponibles (Vergara-Schmalbach, Amézquita, \& Máza, 2008).

Es importante recordar que los escenarios no son una estrategia (Ogilvy \& Schwartz, 1998), más bien, son una herramienta alternativa que permite aclarar el panorama de una organización en el largo plazo, para así direccionar de mejor forma los esfuerzos. Para el diseño de los escenarios se recomienda tener en cuenta 4 grandes agentes de cambios en largo plazo: tecnología, desarrollo de la sociedad, fuerzas económicas globales y efectos políticos, que pueden ser aplicados en cualquier contexto (Lekeas, 1999). El diseño de los escenarios aplicados a la formulación de políticas urbanas, permite confrontar los agentes de cambios en el largo plazo para evaluar la situación y destino de las grandes infraestructuras neurálgicas, en beneficio del desarrollo de una ciudad, región o nación (Zegras, Sussman, \& Conklin, 2004). La aplicación de los escenarios en el sector público, difiere de lo privado, en la complejidad de las decisiones que se toman y la confluencia de variables y grupos de interés que identifican sus problemáticas. Para ello, se debe tener en cuenta en el desarrollo de una metodología de escenarios, el análisis de los factores políticos (sociales, ambientales, económicos), la experticia y experiencia de los líderes del proceso, los niveles de involucración de los grupos de interés en el ejercicio, la selección correcta y la flexibilidad de la metodología (Volkery \& Ribeiro, 2009).

No obstante, en la literatura no se evidencia un esquema de resultados similares en la formulación de escenarios para problemáticas símiles, dado que los contextos e intereses varían de una situación a otra (Ogilvy \& Smith, 2004). En el caso particular de los centros de mercados, la tendencia actual sugiere la desaparición de los mismos, siendo reemplazados por comercios privados, sectorizados y con tamaños ajustados a pequeños grupos de interés (Zade, 2009). Los 4 agentes de cambios, anteriormente mencionados, permean en decisiones sobre adquisición de tecnología, inversión en infraestructura, la reforma de políticas públicas y la gestión ambiental. Para Gonzalez-Feliu, Basck \& Morganti (2003), estas estrategias se traducen en estimaciones monetarias, trasladando la problemática en cómo se deberán adquirir los nuevos recursos que dan viabilidad a los nuevos proyectos que se generen a partir de los escenarios propuestos. Para ello, proponen tres alternativas de financiación: la autofinanciación, los subsidios y los créditos públicos.

\subsection{El método SEARCH para la planeación por escenarios}

Para Kahn (2009) predecir el futuro es una tarea difícil, que depende en gran parte, de entender el presente y el pasado, y de la aplicación de un ejercicio de imaginación y análisis colectivo de expertos, cuyo 
producto es equivalente a una narración hipotética de una serie de eventos (Go \& Carroll, 2004; Kahn, 2009). El objetivo final es apoyar el proceso en la toma de decisiones, excluyendo el cálculo y uso de las probabilidades (Millett, 2009). El método propuesto por Kahn (2009) integró el análisis de la situación en detalle y la valoración de un panel de expertos.

Manteniendo el esquema original del método propuesto por Kahn, el método SEARCH (Sapio, 1995) se convierte en una propuesta independiente (Vergara-Schmalbach, Fontalvo \& Morelos, 2012a), que combina elementos cuantitativos y cualitativos para el diseño de los escenarios. Este método involucra 17 actividades (entre secuenciales y paralelas) (Pagani, 2009) a partir de una caracterización en variables (clasificadas como constantes, predecibles e inciertas) de la problemática o situación analizada. Los principios del método SEARCH se enfocan según Pagani (2009) en: Un evento puede ser afectado o impactado por otros eventos; los expertos son los encargados de valorar la posibilidad de ocurrencia y el impacto generado por cada evento; un grupo de eventos conforman un subescenario, y estos a su vez, recrean los escenarios; la programación matemática y simulación son empleadas para encontrar el escenario con mayor probabilidad de ocurrencia.

\section{Planteamiento de la problemática}

El mercado de Bazurto de la ciudad de Cartagena, fue creado el 22 de enero de 1978 y funge como una plaza de mercado centralizada (Universidad Tecnológica de Bolívar, 2009b), que presta sus servicios de venta de víveres (principalmente), donde converge una esfera pública simbólica y física (Díaz, 2012). El mercado, hoy en día, representa la oportunidad de trabajo disponible para muchas personas (donde un porcentaje significativo de la población Afrodescendiente se encuentra laborando allí) (Correa et al., 2011), proveyendo más de 35.000 puestos de trabajo (Universidad Tecnológica de Bolívar, 2009a), a pesar que fue diseñada para albergar solo 1200 (Transcaribe S.A., 2011). Su desbordado crecimiento alcanza a afectar negativamente su entorno (Ávila, 2007). Dicho efecto se traduce en: inseguridad, incremento del empleo informal, insalubridad, daños en el ecosistema del entorno, tráfico y malestar ge- neral de los habitantes del sector. A través del análisis de diversos estudios (referenciados en la siguiente fase), realizados sobre el mercado de Bazurto y de acuerdo a la metodología o referentes teóricos establecidos, se definieron tres factores clave: medio ambiente, economía y sociedad.

- Factor Medio Ambiente: En 1904, se construyó el mercado de Getsemaní (Plaza que antecedió al actual mercado de Bazurto) (Meisel-Roca, 1999), que en sus inicios gozaba de una excelente infraestructura donde todos sus puestos estaban organizados por sectores que se encargaban de actividades específicas, (sector de carnes, sector de granos, y otras mercancías) lo cual lograba generar un ambiente agradable entre aquellos que se encontraban laborando o mercando en sus instalaciones; pero este panorama no duro mucho tiempo, pues entre los años de 1916 y 1917 se comenzaron a presentar los primeros problemas de contaminación e insalubridad (Gaceta Municipal, 1917).

En 1960, cuando iniciaron las protestas por las condiciones en que había caído el mercado público de Getsemaní, con un problema eminente de contaminación y crecimiento de la población que se acentuaba en el sector, dieron pie al traslado oficial en 1978 al sector Las Quintas (Bazurto) (Fonseca-Castillo, 2010). De esta idea nació un mercado sectorial denominado Pedro A. Salazar (conocido también como mercado de Santa Rita), hoy con notables condiciones de deterioro (Clavijo, 2012). Esta historia se vuelve a repetir en la actualidad, con el mercado de Bazurto, donde las condiciones de insalubridad y afectación al medio ambiente, replantean su vigencia actual. Ricardo Chica (2011), comenta que este referente urbano en su época, generó un avance y progreso en la sociedad ambientando una sensación de optimismo en sus habitantes.

En un estudio realizado sobre la ciénaga La Quinta (cuerpo de agua que recibe los residuos del mercado de Bazurto) durante los años 2009 y 2010, arrojó que ciertos contaminantes en el agua sobrepasaban los límites admisibles para preservación de flora y fauna en aguas marinas (Tirado et al., 2011). El mercado de Bazurto es el punto de mayor contaminación en la ciudad de Cartagena (Contraloría Distrital 
de Cartagena de Indias, 2011), donde el control de los residuos sólidos es insuficiente ante la cantidad de basura generada (Contraloría Distrital de Cartagena de Índias, 2009).

- Factor Económico: El mercado de Bazurto es sin duda alguna, uno de los centros más importantes de empleo en la ciudad (Yánez-Contreras \& Acevedo, 2010). A pesar de estar diseñado originalmente para albergar 1200 puestos, hoy se contabilizan más de 35.000 empleos (y con la tendencia de seguir aumentando), beneficiando no solo a los habitantes de la ciudad, sino también a los municipios aledaños, que ven en el mercado una oportunidad para comercializar sus productos. La mayoría de las personas que laboran en la plaza poseen condiciones financieras desfavorables (Cuadro-Guzman, 2005). Dada la limitación del espacio, el comercio informal aprovecha cualquier espacio disponible para ubicar un punto de venta (428 vendedores informales ocupan parte de las vías de transito normal en la avenida principal de la ciudad Pedro de Heredia) (Transcaribe S.A., 2009). El mercado funciona como una estructura centralizada donde el $80 \%$ de la población se abastece, una zona sobrepoblado y que carece de los servicios públicos básicos (Universidad Tecnológica de Bolívar, 2009b).

- Factor sociedad: El mercado de Bazurto es un lugar donde confluyen muchos habitantes (sin distinción de género, etnia o estrato), donde se observa la cotidianidad de la ciudad (Bolaños et al., 2009; Sanz, 2011). La confluencia de miles de personas y las características socioeconómicas del lugar, generan efectos medio ambientales, confinamiento y condiciones de inseguridad (acompañados de prostitución, extorsión, explotación laboral, drogas y demás perjuicios), que han convertido la plaza en lunar negro para la sociedad cartagenera (Distrito de Cartagena de Indias, 2010; Barrios-Tous, 2011). Esta problemática también se dio en los años 60's, cuando el mercado de Getsemaní se había vuelto un problema por su crecimiento incontrolable, causando congestión y caos en las vías cercanas, y el surgimiento de actividades ilícitas como la prostitución y el contrabando. Gustavo Lemaitre Román, en sus columnas "Mirando por la Rendija" del periódico El Universal, y firmando bajo el seudónimo de Panoptes, adelantó una intensa campaña para erradicar el

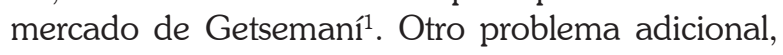
es el tráfico ocasionado por el elevado tránsito vehicular, la invasión del espacio público y la estrechez de la vía. En 1948, el Plan Vial definía como núcleo vial principal la zona de Bazurto, para enlazar todas las líneas de distribución de tráfico y de comunicación urbana (Distrito de Cartagena, 2001).

\section{Metodología}

El presente artículo, está basado en el desarrollo y conclusión de una investigación descriptiva-propositiva, cuyo objetivo principal se constituyó en el diseño de escenarios para el mercado de Bazurto de la ciudad de Cartagena de Indias, aplicando el método de planeación por escenarios (visionando un horizonte de tiempo de 15 años). Manteniendo el esquema original del método SEARCH (ver Ilustracion 1), se estableció una simplificación de actividades, en relación a tratar cada evento identificado (a partir de la descomposición del sistema o problema en variables) como un escenario, dada la abstracción y complejidad de los casos analizados. El método SEARCH es idóneo para el presente caso, al considerar un número amplio de variables (por factores) e incluir componentes de valoración cualitativa realizadas por un panel de expertos en varios momentos del desarrollo del método.

Las fases desarrolladas en esta investigación fueron: Fase 1 - Identificación de los factores clave (definición del sistema, descomposición del sistema en factores y variables clave, y formulación de sub-escenarios); Fase 2 - Valoración de sub-escenarios (selección del panel de expertos y evaluación de los sub-escenarios por el panel de experto); Fase 3 - Cálculo y Formulación de escenarios (Selección de los sub-escenarios más probables, valoración de la compatibilidad entre pares de escenarios, análisis de impacto cruzado y cálculo de probabilidades); y Fase 4 - Análisis, redacción y validación de escenarios (redacción de escenarios y validación de los escenarios).

1 Fuente: Entrevista realizada a José Henrique Rizo Pombo el 24 de enero de 2011. 
Ilustración 1. Esquema metodológico del método SEARCH²

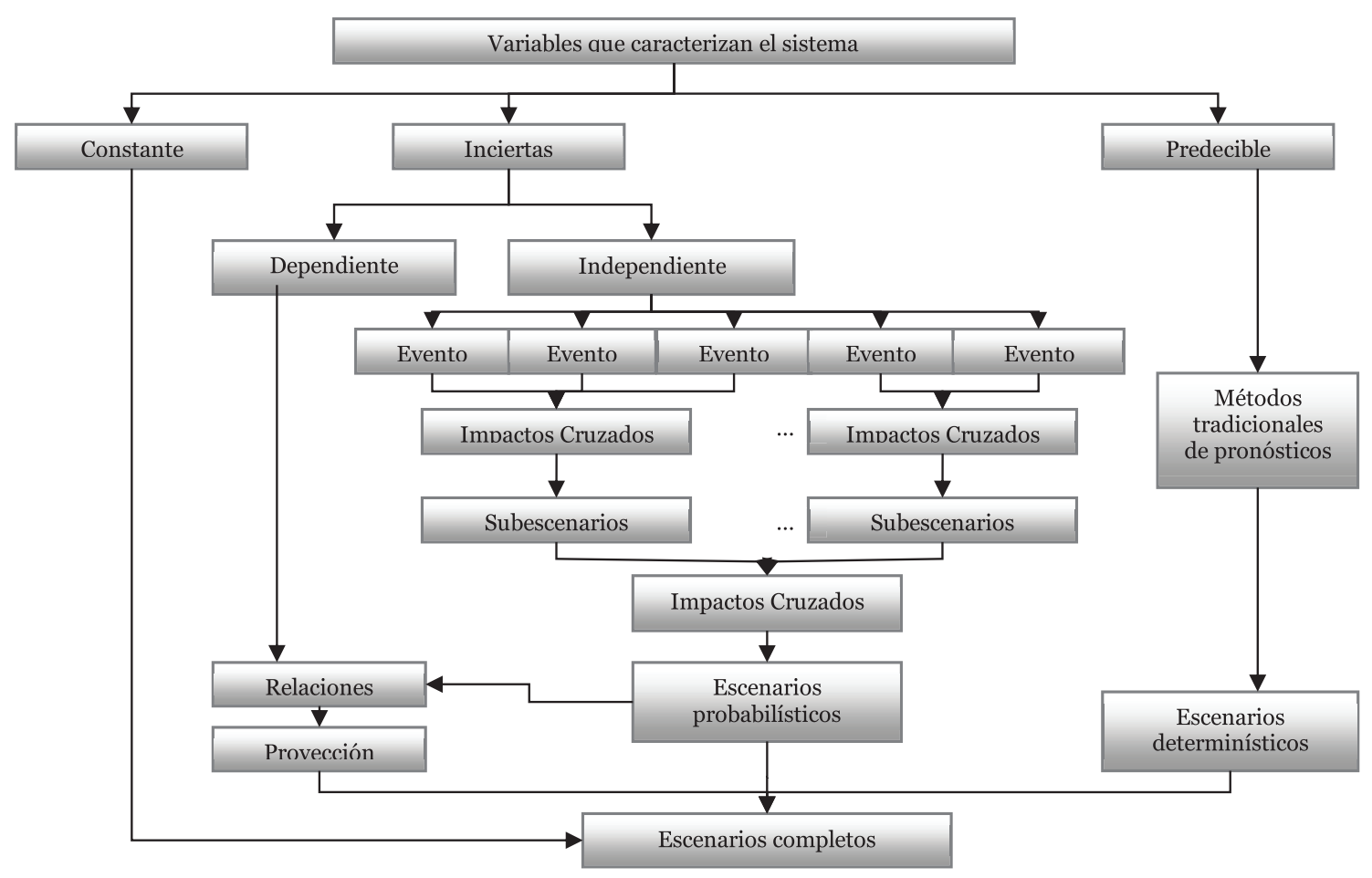

\subsection{Clasificación de las variables}

Las variables denominadas constantes, se mantienen estables en el tiempo; las variables predecibles pueden ser empleadas para identificar tendencias y las variables inciertas, son gobernadas por eventos símiles a comportamientos aleatorios (Vergara-Schmalbach et al., 2012b). De los factores identificados en el planteamiento de la problemática, se relacionaron un total de 9 variables clasificadas en previsible, constantes e inciertas (Tabla 1).

\subsection{Constitución del panel de expertos}

Un análisis bibliográfico exhaustivo realizado por Michel Keenan (2007), sobre los métodos empleados para ayudar a conocer el fututo, encontró que la consulta a un panel de expertos es una herramienta am- pliamente usada (el $52 \%$ de los 755 casos revisados por keenan se centran exclusivamente en el panel de expertos como herramienta general de pronóstico, de los cuales en el $40 \%$ de los casos, se recurre al panel para valorar o construir escenarios). Derivado de un análisis propio de los métodos que requieren de la participación de un panel de expertos en proceso de diseño de los escenarios, se concluye que el panel de expertos debe estar conformado por un equipo humano interdisciplinario (Weimer-Jehle, 2008), conocedores del problema, y en clientes y/o consumidores (Lindgren \& Bandhold, 2003), con capacidad de plantearse o encarar un futuro (Godet, 2000) o de juzgar posibles futuros (Sapio, 1995).

Para esta investigación, y siguiendo las pautas de selección establecidas, se seleccionaron 45 personas para conformar el panel de expertos, cuyas carac-

2 Fuente: Traducción realizada por los autores del método SEARCH de Sapio (1995). 
Tabla 1. Clasificación de las variables ${ }^{3}$

\begin{tabular}{|c|c|c|}
\hline Factor & Variables & Tipo de variables \\
\hline \multirow{3}{*}{ Medio ambiente } & Contaminación & $\begin{array}{c}\text { Constante } \\
\text { Incierta } \\
\end{array}$ \\
& Salud pública & Constante \\
\hline \multirow{2}{*}{ Económico } & Emejo de residuos sólidos & Previsible \\
& Servicios públicos & Previsible \\
& Comercio & Previsible \\
\hline \multirow{2}{*}{ Sociedad } & Seguridad & Constante \\
& Riqueza social & Constante \\
& Tráfico & Constante \\
\hline
\end{tabular}

terísticas se enfocaron en el conocimiento suficiente de la situación del mercado de Bazurto. El panel fue integrado por (ex) miembros del gobierno, investigadores, comerciantes y habitantes de la zona aledaña.

\section{Desarrollo}

A partir de la interrelación de las variables se obtuvo 9 sub-escenarios ( 3 por cada factor) como se muestran en la siguiente Tabla 2.

Tabla 2. Lista de sub-escenarios ${ }^{4}$

\begin{tabular}{|c|c|}
\hline Nombre & Factor Clave \\
\hline $\begin{array}{c}\text { S1. Infraestructura para el tratamiento } \\
\text { de los residuos sólidos } \\
\text { S2. Mitigación del impacto ambiental } \\
\text { S3. Traslado del mercado }\end{array}$ & Medio Ambiente \\
\hline S4. Formalización de vendedores & \\
S5. Sectorización del mercado & Económico \\
S6. Traslado de vendedores & \\
\hline S7. Plan de seguridad & \\
S8. Enfoque cultural & Sociedad \\
S9. Rehabilitación vial & \\
\hline
\end{tabular}

Dichos sub-escenarios fueron valorados por el panel de expertos bajo una escala numérica de 1 a 5 , donde 1 corresponde a un escenario improbable y 5 , a un escenario probable. En la Tabla 3, se puede observar el promedio de las valoraciones dadas por el panel de expertos.

Tabla 3. Promedio de valoración de los sub-escenarios ${ }^{5}$

\begin{tabular}{|c|c|c|c|}
\hline Sub-escenarios & $\begin{array}{c}\text { Calificación } \\
\text { promedio }\end{array}$ & Probabilidad & Resultado \\
\hline S1 & 4.1 & $82,40 \%$ & Probable \\
S2 & 4.3 & $86,40 \%$ & Probable \\
S3 & 4.9 & $98,60 \%$ & Probable \\
S4 & 3.9 & $78,00 \%$ & Poco probable \\
S5 & 4.6 & $92,70 \%$ & Probable \\
S6 & 4.7 & $94,00 \%$ & Probable \\
S7 & 3.8 & $76,20 \%$ & Poco probable \\
S8 & 4.2 & $83,80 \%$ & Probable \\
S9 & 4.5 & $90,40 \%$ & Probable \\
\hline
\end{tabular}

\footnotetext{
Fuente: Elaboración propia.

Fuente: Elaboración propia.

Fuente: Elaboración propia.
} 
Tabla 4. Matriz de impacto cruzado ${ }^{6}$

\begin{tabular}{|c|c|c|c|c|c|c|c|c|c|}
\hline & S1 & S2 & S3 & S4 & S5 & S6 & S7 & S8 & S9 \\
\hline S1 & & & & 1 & 0 & 0 & 0 & 2 & 0 \\
\hline S2 & & & & 3 & 0 & 3 & 0 & 2 & 0 \\
\hline S3 & & & & 1 & 3 & 3 & 2 & -1 & 2 \\
\hline S4 & 2 & 3 & 2 & & & & 3 & 2 & 0 \\
\hline S5 & 3 & 2 & 3 & & & & 3 & -1 & 2 \\
\hline S6 & 1 & 3 & 3 & & & & 3 & -1 & 2 \\
\hline S7 & 0 & 1 & 0 & 2 & 1 & 1 & & & \\
\hline S8 & 1 & 3 & 2 & 3 & 1 & 2 & & & \\
\hline S9 & 2 & 1 & 2 & 0 & 1 & 0 & & & \\
\hline
\end{tabular}

Tabla 5. Escala Ordinal para la valoración causal de los sub-escenarios ${ }^{7}$

\begin{tabular}{|c|l|}
\hline Intensidad del impacto & \multicolumn{1}{|c|}{ Descripción } \\
\hline+3 & Influencia directa promotora fuerte \\
\hline+2 & Influencia directa promotora moderada \\
\hline+1 & Influencia directa promotora débil \\
\hline 0 & Sin influencia \\
\hline-1 & Influencia directa restrictiva débil \\
\hline-2 & Influencia directa restrictiva moderada \\
\hline-3 & Influencia directa restrictiva fuerte \\
\hline
\end{tabular}

Con el uso del software Scenario Wizard Basic 3, se obtuvo un total de 2 combinaciones que presentaron una consistencia fuerte (Tabla 6).

Tabla 6. Escenarios con calificaciones más altas de impacto $^{8}$

\begin{tabular}{|l|l|}
\hline \multicolumn{1}{|c|}{ Escenario no. 1} & \multicolumn{1}{c|}{ Escenario no. $\mathbf{2}$} \\
\hline Puntaje de impacto total: 12 & Puntaje de impacto total: 16 \\
\hline A. Medio amb.: S3 & A. Medio amb.: S2 \\
\hline B. Economico: s5 & B. Económico: s4 \\
\hline C. Sociedad: s7 & C. Sociedad: s8 \\
\hline
\end{tabular}

\footnotetext{
Fuente: Elaboración propia.

Fuente: Elaboración propia.

Fuente: Elaboración propia.

Fuente: Elaboración propia.
}

\section{Resultados}

En la Tabla 7, se muestran los 2 escenarios con el cálculo respectivo de su probabilidad de ocurrencia en orden jerárquico (de mayor a menor probabilidad).

Tabla 7. Escenarios con mayores probabilidades de ocurrencia $^{9}$

\begin{tabular}{|c|c|c|}
\hline Escenario & Sub-escenarios & Probabilidad \\
\hline \multirow{2}{*}{1} & S3 & $98,60 \%$ \\
\cline { 2 - 3 } & S5 & $92,70 \%$ \\
\cline { 2 - 3 } & S7 & $76,20 \%$ \\
\hline Probabilidad de ocurrencia & \multicolumn{2}{|c|}{$69,65 \%$} \\
\hline \multirow{2}{*}{ Escenario } & Sub-escenarios & Probabilidad \\
\hline \multirow{2}{*}{2} & S2 & $86,40 \%$ \\
\cline { 2 - 3 } & S4 & $78,00 \%$ \\
\cline { 2 - 3 } & S8 $83,80 \%$ \\
\hline Probabilidad de ocurrencia & \multicolumn{2}{|c|}{$56,47 \%$} \\
\hline
\end{tabular}

En este caso se proponen dos escenarios en líneas contrarias: el primero de ellos apunta al traslado de la plaza, mientras que el segundo escenario propone una estrategia de fortalecimiento e intervención del mismo. La redacción de ambos escenarios se presenta a continuación, contenido validado por el panel de expertos:

- Escenario 1 - Traslado y sectorización del mercado y diseño de un plan de seguridad integral: En el año 2026, la ciudad de Cartagena de Indias D.T. podrá contar con una nueva plaza de mercado y central de abasto, ubicado en la periferia de la ciudad. El antiguo mercado de Bazurto quedaría inactivo, reconstituyendo la zona en condiciones adecuadas para el transito normal de personas, el desarrollo normal del ecosistema y la construcción de opciones de vivienda.

Para evitar la sobrepoblación del nuevo mercado, se desarrollarán nuevas plazas sectorizadas (como el actual mercado de Santa Rita), distribuyendo en gran medida, los puestos de trabajo y el acceso de 
los consumidores. Para mantener el orden, tanto el nuevo mercado central como los mercados sectorizados, se dispondrá del control y vigilancia permanente (privada y pública), evitando la proliferación de la inseguridad, el mal uso de los residuos generados y la afectación desfavorable en las zonas vecinas. Este nuevo orden involucraría, la reglamentación de cada plaza de mercado con relación a la convivencia, comercio, economía y seguridad, en procura del desarrollo socio-sostenible y de la conservación del patrimonio cultural.

- Escenario 2 - Mitigación del impacto ambiental, formalización de vendedores y enfoque cultural: En el año 2026, la ciudad podrá disponer de un renovado mercado de Bazurto, con controles y reglamentaciones adecuadas para el manejo ambiental y mitigación de su impacto. Esta estrategia involucraría el desarrollo de un sistema de gestión ambiental para el tratamiento de los residuos generados, convirtiendo al mercado, en un desarrollo sostenible.

En esta misma línea de ideas, todos los comerciantes ubicados en la plaza estarán debidamente formalizados y capacitados, evitando así la proliferación de nuevos puestos de trabajos informales. El control exhaustivo por parte del gobierno Distrital, sumado a la capacitación e inversión en infraestructura, garantizarán en gran medida el sostenimiento del orden en el sector. Con estrategias de formación permanente y el desarrollo de proyectos culturales, el mercado de Bazurto seguirá siendo un punto de riqueza social en la ciudad. En el año 2026, Bazurto dejará de ser un lunar negro en la ciudad, y pasará a convertirse en una plaza moderna y eficiente, con un fuerte atractivo cultural y turístico.

\section{Discusión y conclusiones}

La problemática tratada en este artículo, considera una situación que parece repetirse en varios países latinoamericanos, como México, Colombia y Bolivia (Pérez 2004; Suárez 2006; Berrocal, 2009; García \& Flores 2011; Gutierrez 2013), donde predominó la hegemonía de un mercado central (situación característica de pequeñas ciudades, municipios o pueblos), y cuyas soluciones han radicado en acciones de intervención y relocalización. En el caso concreto de Colombia, la problemática de las plazas de mercado suelen asociarse a la invasión del espacio público, la contaminación, el empleo informal y la inseguridad (Rodríguez-Reyes, 2009), factores que están presente en el mercado de Bazurto de la ciudad de Cartagena de Indias.

Las plazas de mercados son consideradas como un servicio público amparadas en el artículo 365 de la constitución política colombiana. Estas zonas tienden a actuar como centros donde la comunidad interactúa libremente, abasteciéndolos de los insumos necesarios (por lo general, a personas de escasos recursos). Las plazas son también el destino de muchos productos agrícolas, para aquellos campesinos que no pueden acceder a las grandes superficies comerciales directamente. Para Gillespie et. al. (2008) los mercados públicos están más vigentes que nunca y funcionan como centrales mayoristas que distribuyen víveres en las localidades, con los siguientes beneficios: incrementan la visibilidad de los productores regionales, alientan la diversificación de la producción regional, incuban y soportan pequeños negocios, y crean un espacio para las transacciones económicas y sociales

Por otro lado, una inadecuada gestión administrativa de las plazas de mercado conlleva al deterioro de su infraestructura, la sobrepoblación, la negligencia en el manejo de los residuos y por ende, la afectación negativa de su entorno. Por esta razón, algunas plazas en Colombia están siendo reestructuradas (Suárez, 2006; Rodríguez-Reyes, 2009) o reubicadas (Barragan, 2002). Estas dos acciones fueron visualizadas en los escenarios propuestos para el mercado de Bazurto, siendo la más probable la reubicación de la plaza a otro lugar, estrategia que va en la dirección del sentir de sus habitantes (Darío-Álvarez, 2011) acompañado de la creación de pequeñas plazas sectoriales; no dejando aún de lado el segundo escenario, donde la formalización de vendedores, la mitigación del impacto ambiental y el enfoque cultural podrían evitar que se repitiese el caso del antiguo mercado Getsemaní, donde el traslado a su ubicación actual se convirtió en una solución temporal.

En este artículo se demuestra la aplicabilidad del método de planeación por escenarios como coad- 
yuvante en el proceso de toma de decisiones. Con el análisis de la historia y trayectoria del mercado de Bazurto, los estudios ambientales realizados en la zona, las tendencias económicas, la comparación con casos exitosos y la consulta de expertos, permitieron ejecutar una metodología para el diseño de escenarios plausibles que soportan las estrategias aquí planteadas. El método aquí planteado combinó las herramienta cualitativas (panel de expertos, método de impactos cruzados, análisis documental) y cuantitativas (proyecciones económicas y ambientales, probabilidades), promoviendo una visión holística de la situación.

Se concluye que ciudades como Cartagena, no deben quedarse sin plazas de mercado, justificando su conservación como fuente de riqueza social y venta al detalle de productos a un bajo costo (Zade, 2009). El mercado deberá en primera medida, ser reubicado en una zona adecuada que no afecte drásticamente su nuevo entorno. Si esta estrategia no llegase a cumplirse, la segunda opción se traduce en una intervención estructural.

Con el traslado de Bazurto y creación de nuevas plazas se podrá empezar un nuevo esquema de funcionamiento con el fin de alcanzar la eficiencia locativa, concentrar la demanda, ser el éxito de los negocios locales, ser un lugar de acopio para la comunidad, motivar el empleo, conectar el ambiente urbano y rural; elemento que conforman el nuevo paradigma de los mercados públicos (Moon, 2006). En futuras investigaciones se propone utilizar herramientas para el análisis de sistemas complejos (como la simulación y la dinámica de sistemas), para entender el efecto directo o indirecto entre las distintas variables definidas para el diseño de los escenarios. Las propuestas aquí presentes podrían concretarse en un modelo de desarrollo social, sostenible y de conservación del patrimonio cultural para las plazas de mercado.

\section{Referencias}

Ávila, F. (2007). "Bazurto: Un arroz con mango". En: Noventa y Nueve, 7(1): 20-26.

Barragan, M. (2002). Tutela para reubicar plazas de mercado. En: Periódico El Tiempo, Bogotá D.C.

Barrios-Tous, E. (2011). Bazurto, el mismo problema. En: Periódico El Universal. Cartagena. Disponible: http://www.eluniversal.com. co/cartagena/politica/bazurto-el-mismo-problema
Berrocal, B. (2009). El desaseo es el principal problemas de los mercados. En: Periódico El Universal, Cartagena.

Bolaños, N., Cabrales, C., Cogollo, K., Hernández, J. \& Pérez, L. (2009). Representaciones sociales sobre la ciudad en la Cartagena contemporánea. Cartagena: Universidad de Cartagena.

Börjeson, L., Höjer, M., Dreborg, K., Ekvall, T. \& Finnveden, G. (2006). Scenario types and techniques: Towards a user's guide. En: Futures, 38(7): 723-739.

Bradfield, R., Wright, G., Burt, G., Cairns, G. \& Van Der Heijden, K. (2005). "The origins and evolution of scenario techniques in long range business planning". En: Futures, 37(8): 795-812.

Chermack, T., Lynham, S. \& Ruona, W. (2001). "A Review of Scenario Planning Literature". En: Futures Research Quartely, 17(2): 7-30.

Chica, R. (2011). Memoria Radial Solle: Dinámicas urbanas, música mundo e identidad juvenil en los sectores populares de Cartagena 1975-1985. Cartagena: Editorial Universitaria Universidad de Cartagena.

Clavijo, A. (2012). En Cartagena: "Mercado de Santa Rita se cae a pedazos". En: Periódico El Universal, Cartagena. Disponible: http://www.eluniversal.com.co/cartagena/local/en-cartagenamercado-de-santa-rita-se-cae-pedazos-77376

Contraloría Distrital de Cartagena de Indias. (2011). Estado de los Recursos Naturales y del Medio Ambiente 2008-2011. Cartagena: Contraloría Distrital de Cartagena de Indias.

Contraloría Distrital de Cartagena de Indias. (2009). Sistema de aseo público del Distrito de Cartagena de Indias - Vigencia 2007 2008. Cartagena: Contraloría Distrital de Cartagena de Indias.

Cornelius, P., Van de Putte, A. \& Romani, M. (2005). "Three Decades of Scenario Planning in Shell”. En: California Management Review, 48(1): 92-109.

Correa, G., Pérez, A., Viveros, V., Morales, M. \& Flórez, J. (2011). Precario pero con trabajo: iotros están peor! Medellín: Ediciones Escuela Nacional Sindical.

Cuadro-Guzman, E. (2005). Alternativas viables para convertir la Relocalización de Comerciantes Ocupantes del Espacio Público, por motivo de Macroproyectos de Infraestructura, en oportunidades de mejoramiento socioeconómico. II Foro Técnico Regional sobre Reasentamiento de Población. Bogotá, 25 - 27 Mayo.

Darío-Álvarez, R. (2011). Traslado del mercado alegra a sus vecinos. En: Revista El Universal, Cartagena.

Díaz, L. (2012). "Emergentes concepciones de ciudad patrimonio a partir del Mercado de Bazurto de Cartagena de Indias: de la ciudad perfecta a la ciudad diversa". En: Memorias: Revista Digital de Historia y Arqueología desde el Caribe, 9(18): 219-237.

Distrito de Cartagena (2001). Decreto N0. 0977 de 2001. Colombia: Distrito de Cartagena

Distrito de Cartagena de Indias. (2010). Política Pública para la infancia y la adolescencia en el Distrito Turístico y Cultural de Cartagena de Indias. Cartagena: Distrito de Cartagena de Indias.

Fonseca-Castillo, G. (2010). De Getsemaní a Bazurto. En: Periódico El Universal, Cartagena. Disponible: http://www.eluniversal.com. $\mathrm{co} /$ suplementos/dominical/de-getsemani-bazurto

Gaceta Municipal (1917). Cartagena: Alcaldía Municipal de Cartagena

García, C. \& Flores, I. (2011). Mercados públicos municipales: el caso del mercado Benito Juárez de Ciudad Ixtepec, Oaxaca, México, (2009-2010). En: Debates Latinoamericanos, 3(9): 1-19.

Gillespie, G., Hilchey, D., Hinrichs, C. \& Feenstra, G. (2008). "Farmer's markets as keystone in rebuilding local and regional 


\section{LA PLANEACIÓN POR ESCENARIOS APLICADA SOBRE POLÍTICAS URBANAS: EL CASO DEL MERCADO CENTRAL DE CARTAGENA (COLOMBIA)}

food Systems". En: Remaking the North American Food System: Strategies for Sustainability. Nebraska: U of Nebraska Press.

Go, K., \& Carroll, J. (2004). "The blind men and the elephant". En: Interactions, 11(6): 44-53.

Godet, M. (2000). La caja de herramientas de la prospectiva estratégica. Paris: Laboratoire d'Investigation Prospective et Stratégique.

Godet, M. (2006). Creating Futures: Scenario Planning as a Strategic Management Tool (2da ed.). London: Economica.

Gonzalez-feliu, J., Basck, P., y Morganti, E. (2003). "Urban logistics solutions and financing mechanisms: a scenario assessment analysis". En: European Transport, 54(11): 1-16.

Gutierrez, C. (2013, Julio 13). Los conflictos en el interior de las plazas. En: Periódico El Espectador, Bogotá D. C.

Kahn, H. (2009). The Essential Herman Kahn. New York. United Kingdom: Lexington Books.

Keenan, M. (2007). Combining Foresight Methods for Impacts. NISTEP 3rd International Conference on Foresight. Tokio, 19 y 20 noviembre.

Lekeas, G. (1999). Scenario Planning, Formulation, Implementation and Effects: A review of best theory and practice. Warwick: University of Warwick.

Lindgren, M. \& Bandhold, H. (2003). Scenario Planning: The link between future and strategy. New York: Palgrave Macmillan.

Meisel-Roca, A. (1999). Cartagena 1900-1950: A remolque de la Economía Nacional. Cartagena: Banco de la República.

Millett, S. M. (2009). "Should Probabilities Be Used with Scenarios?" En: Journal of futures Studies, 13(4): 61-68.

Moon, J. R. (2006). Public Markets and Community Health: An Examination. Columbia: The Ford Foundation.

Ogilvy, J. \& Schwartz, P. (1998). Plotting Your Scenarios. Emeryville: Global Business Network (GBN)

Ogilvy, J. \& Smith, E. (2004). "Mapping Public and Private Scenario Planning: Lessons from regional projects". En: Development, 47(4): 67-72.

Pagani, M. (2009). "Roadmapping $3 G$ mobile TV: Strategic thinking and scenario planning through repeated cross-impact handling". En: Technological Forecasting and Social Change, 76(3): 382-395.

Pérez, L. (2004, Noviembre 24). En riesgos mercados públicos del DF. En: Periódico EsMas. México D. F.

Porter, M. E. (1998). Competitive Advantage: creating and sustaining superior performance. New York: Free Press.

Rodríguez-Reyes, M. (2009). La función de los mercados mayoristas en los centros urbanos de Colombia . Bogotá: FAO - Organización de las Naciones Unidas para la Agricultura y la Alimentación.

Sanz, M. (2011). Fiesta de Picó: Champeta, espacio y cuerpo en Cartagena, Colombia. Tesis de Grado. Bogotá, Universidad del Rosario.

Sapio, B. (1995). "SEARCH (Scenario evaluation and analysis through repeated cross impact handling): a new method for scenario analysis with an application to the Videotel service in Italy". En: International Journal of Forecasting, 11(1): 113-131.

Schoemaker, P. J. (1995). "Scenario planning: a tool for strategic thinking”. En: Sloan Management Review, 36(2): 25-40.

Suárez, O. (2006). Establecimiento de los lineamientos para la gestión ambiental y sanitaria de las plazas de mercado del Distrito Capital. Bogotá D.C: Alcaldía Mayor de Bogotá.
Tirado, O., Manjarrez, G. \& Díaz, C. (2011). "Caracterización Ambiental de la Cienagas de La Quinta localizada en Cartagena de Indias, Colombia, 2009 - 2010”. En: Revista U.D.C.A Actualidad y Divulgación Científica, 14(2): 131-139.

Transcaribe S.A. (2009). Plan para los cocupantes del Espacio Público ubicados en el Centro y en el Mercado de Bazurto. Cartagena: Transcaribe S.A.

Transcaribe S.A. (2011). Optimización del diseño del Plan de Manejo Ambiental y Gestión Social para la Construcción del Tramo Bazurto - Popa. Cartagena: Transcaribe S.A.

Universidad Tecnológica de Bolívar. (2009a). Retos y oportunidades para la acción en el Mercado de Bazurto: Análisis de cuatro cadenas de abastecimiento e implicaciones para la ciudad de Cartagena. Cartagena: Edicciones Universidad Tecnológica de Bolívar

Universidad Tecnológica de Bolívar (2009b). Mercado de Bazurto: ¿Cómo Gestionar el Cambio? Cartagena: Ediciones Universidad Tecnológica de Bolívar.

Vergara-Schmalbach, J. C., Amézquita, J. \& Máza, F. (2008). "Diseño y análisis de escenarios a partir de la caracterización de las cadenas productivas mediante modelos de redes". En: Revista Panorama Económico, 16(1): 73-89.

Vergara-Schmalbach, J. C., Fontalvo, T. J. \& Máza, F. (2010). "La planeación por escenarios: Revisión de conceptos y propuestas metodológicas". En: Prospectiva, 8(2): 21-29.

Vergara-Schmalbach, J. C., Fontalvo, T. J. \& Morelos, J. (2012a). Aplicación del Método de Decisión Multicriterio UTASTAR para la Selección de Portafolios de Inversión. Global conference on Business and Finance. 23-25 de Mayo.

Vergara-Schmalbach, J. C., Fontalvo, T. \& Morelos, J. (2012b). Aplicación de la Planeación por Escenarios en Unidades Académicas: Caso Programa de Administración Industrial. En: Revista Escenarios, 10(1): 40-48.

Vergara-Schmalbach, J. C., Máza, F. \& Fontalvo, T. J. (2010). "Futurología: origen, evolución y métodos". En: Palobra, 11(1): 218-229.

Volkery, A. \& Ribeiro, T. (2009). "Scenario planning in public policy: Understanding use, impacts and the role of institutional context factors". En: Technological Forecasting and Social Change, 76(9): 1198-1207.

Wack, P. (1985). Scenarios: Uncharted Waters Ahead. En: Harvard Business Review, 63(5): 73-89.

Weimer-Jehle, W. (2006). Cross-impact balances: A system-theoretical approach to cross-impact analysis. En: Technological Forecasting and Social Change, 73(4): 334-361.

Weimer-Jehle, W. (2008). "Cross-impact balances". En: Physica A: Statistical Mechanics and its Applications, 387(14): 3689-3700.

Yánez-Contreras, M. \& Acevedo, K. (2010). "Determinantes de la estructura espacial del empleo en Cartagena". En: Sociedad y Economía, 19(1): 179-204.

Zade, J. C. (2009). Public Market Development Strategy: Making the Improbable Posible. Master Thesis. Massachusetts, Massachusetts Institute of Technology.

Zegras, C., Sussman, J. \& Conklin, C. (2004). "Scenario Planning for Strategic Regional Transportation Planning". En: Journal of Urban Planning and Development, 130(1): 2-13. 
